\title{
Behavioral Assessment
}

National Cancer Institute

\section{Source}

National Cancer Institute. Behavioral Assessment. NCI Thesaurus. Code C81234.

Assessment of individual responses to age appropriate stimuli. 